\title{
Rotational Legged Locomotion
}

Damian M. Lyons

Fordham University

Kiran Pamnany

Fordham University

Follow this and additional works at: https://fordham.bepress.com/frcv_facultypubs

Part of the Robotics Commons

\section{Recommended Citation}

Lyons, Damian M. and Pamnany, Kiran, "Rotational Legged Locomotion" (2005). Faculty Publications. 32.

https://fordham.bepress.com/frcv_facultypubs/32

This Article is brought to you for free and open access by the Robotics and Computer Vision Laboratory at DigitalResearch@Fordham. It has been accepted for inclusion in Faculty Publications by an authorized administrator of DigitalResearch@Fordham. For more information, please contact considine@fordham.edu. 


\title{
Rotational Legged Locomotion
}

\author{
Damian M. Lyons and Kiran Pamnany \\ Robotics and Computer Vision Laboratory \\ Department of Computer and Information Science \\ Fordham University \\ Bronx NY 10458 \\ $\{d l y o n s$, pamnany\}@cis.fordham.edu
}

\begin{abstract}
In this paper we presented a novel, agile robot mechanism, which we call a rotopod, which combines aspects of wheeled and legged locomotion. A general description of how a tripedal rotopod can be made to step, rotating the mechanism about one leg, and moving the center of the mechanism, is presented. The concept of a gait for this mechanism is defined, and is used to show how extremely agile the mechanism can be. Specific resistance is employed as a way to explore the relative efficiency of this mechanism versus a wheel. Finally, we describe our first prototype rotopod and report on experiments conducted to characterize stepping.
\end{abstract}

Index Terms - Mobile robots, Legged locomotion, Tripedal, Energy-efficient.

\section{INTRODUCTION}

$\mathrm{T}$ he majority of the current generation of mobile robots are wheeled vehicles. Wheels are efficient, easy to control and well understood. However, there are terrains that are not easily traversed with wheels, and much effort has been expended to explore legged robot mechanisms that can, for example, step over obstacles and on extremely rough terrain, can ascend and descend stairway terrain, and can give a smoother ride over rough ground. Legged vehicles tend to be more complicated mechanically than wheeled vehicles and their efficiency lower since they must lift as well as propel the vehicle. It is understandable therefore that many efforts have been made to combine wheeled and legged locomotion, e.g., Halme et al. [7].

This paper introduces a robot mechanism which combines the features of wheeled and legged locomotion in an unusual way. This robot has the advantage of legged locomotion in stepping its 1-DOF legs over objects, but its drive mechanism is a rotating reaction mass that rotates the robot, in a controllable fashion, around each of its legs, similar to a rotating wheel. The mechanism has the potential to transfer the energy from the rotating reaction mass in an efficient manner to the legs, effecting a spinning forward motion. Such a device could have applications as a small fast-moving and extremely agile mobile sensor platform. A large quantity of small versions of this mechanism could be dropped to map and explore an area, or larger versions could be used as for unmanned forward reconnaissance. This paper presents our first steps in designing, constructing and evaluating this class of robot mechanism which we call a rotopod.

In the next section, we briefly overview previous work in legged locomotion as it relates to the rotopod mechanism. Section III introduces the general rotopod mechanism, discusses locomotion style, gaits, and efficiency. Section IV presents our current prototype robot, a simple rotopod and its control hardware and software. Section V presents results on the characterization of the movement of the mechanism. Section VI reports our conclusions and plans for future rotopod work.

\section{LITERATURE REVIEW}

Several multi-legged mechanisms have been described in the literature, e.g., Dante [3], Attila [2], Sony's AIBO, NTU's multifunctional quadruped [8]. These devices have an important stability advantage, since at all times three legs can be lowered to a stable tripod while other legs move. However, energy efficiency is a key issue with this style of locomotion.

Raibert [13, 12] investigated an energy efficient hopping locomotion style in one-legged up to four-legged mechanisms. The RHex robot [1] is a hexapod in which the 'legs' are replaced by are rotating flexible beams. Running consists of rotating the legs in one of a large variety of gaits. Whegs [11], a series of robots inspired by studies of cockroach locomotion, have legs that are three-spoked, rimless 'wheels'. More recently there has been interest in bipedal robots, e.g., Honda's ASIMO. Bipedal locomotion can potentially be very efficient; a bipedal motion, rolling from leg to leg, is very similar to a wheel rolling [6].

A small number of three-legged designs have also been explored, including LIBRA [5], the spring-attenuated tripod platform of Badreddin [4], the Harunori tripedal robot [9]. These mechanisms locomote by using the DOF of each leg to push the body along. Our three legged design is very different. The drive mechanism is a rotating reaction mass that causes the robot to spin around each leg. In the slow, step by step rotation that will be described in this paper, this mode of locomotion is relatively inefficient. However, the mechanism has the potential to do continuous rotation, producing energyefficient legged locomotion by rolling in a manner similar to a single wheel device such as the Gyrover [14]. 


\section{A Rotational WaLKing MechanisM}

\section{A. Mechanism.}

A general tripedal rotopod mechanism consists of three legs arranged in a tripod configuration. Each leg is equipped with a single translational degree of freedom, so that the leg length for each leg can be varied between $\ell$ and $\ell_{\mathrm{e}}=\ell+\delta$. A rotating joint is placed at the apex of the tripod. The joint rotates an armature of length $r$ at the end of which is placed a reaction mass $m_{r}$. This general tripedal rotopod is illustrated in Fig. 1.

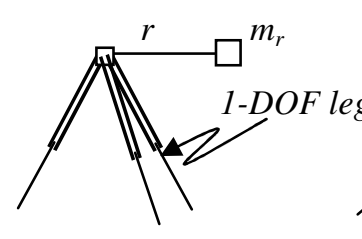

(a) Side View

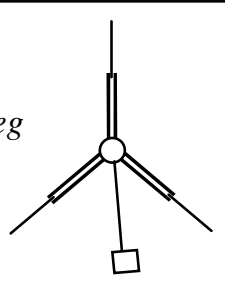

(b) Top View
Figure 1: General Tripedal Rotopod

When all three legs are extended at length $\ell_{\mathrm{e}}$ the plane of rotation of the apex joint is parallel to the ground plane. If one of the legs is retracted to length $\ell$, then the plane of rotation of the apex joint is tilted. It tilts around the line joining the endpoints of the legs opposite the retracted leg. If the angle each leg makes with the vertical centerline is $\beta$, then the ground distance between the projection of the apex and a leg endpoint is $\ell_{e} \operatorname{Sin} \beta$. If all three legs are positioned equally around the apex, then the ground distance from a leg endpoint to the midpoint of the line joining opposite leg endpoints is $1.5 \ell_{e} \operatorname{Sin} \beta$. If one leg retracts a distance $\delta$, then the leg drops a vertical distance $\delta \operatorname{Cos} \beta$. The resulting angle $\alpha$ about the line joining opposite legs is

$$
\tan \alpha=\frac{\delta \operatorname{Cot} \beta}{\left(1.5 l_{e}-\delta\right)}
$$

The weight of the platform and the weight of the reaction mass are chosen so that the platform is balanced when the legs are fully extended. The rotating reaction mass applies a force $f_{r}$ which pulls on the apex. The platform weight counters this.

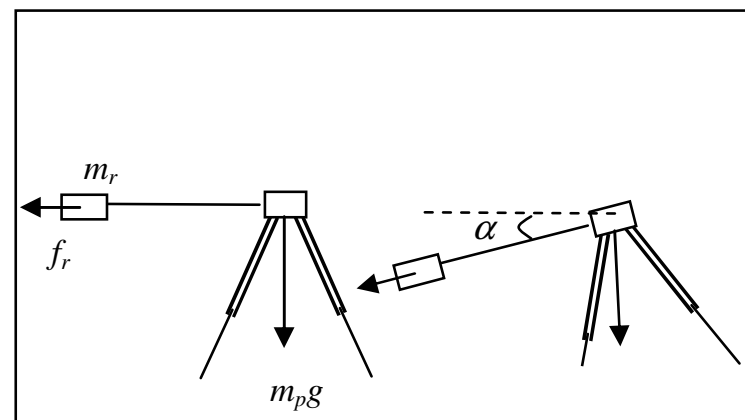

(a) balanced

(b) unbalanced

Figure 2: Quasi-static model of balance

$$
f_{r}=m_{r} \omega^{2} r \quad f_{w}=m_{p} g
$$

where $\omega$ is the angular velocity of the reaction mass. Resolving these forces perpendicular to the retracted leg, and equating the moments, then, in the balanced case (Fig. 2(a)), we have that:

$$
l_{e} f_{r} \operatorname{Sin} \beta=l_{e} f_{w} \operatorname{Cos} \beta
$$

\section{B. Stepping the Mechanism.}

However, when the platform is tilted, this balance is changed. There is now a smaller angle between the lower leg and $f_{w}$ meaning that the contribution of the weight in balancing the mechanism is lessened. The components of forces can be resolved perpendicular to the retracted leg and the moments summed. Let the net moment be $M_{n e t}=l_{e} f_{b}$, where we will call the net unbalance force $f_{b}$. When this is sufficiently large, the mechanism will rotate around the endpoint of the retracted leg, raising the other legs off the ground. Fig. 3 shows a graph of the growth of $f_{b}$ versus tilt in degrees.

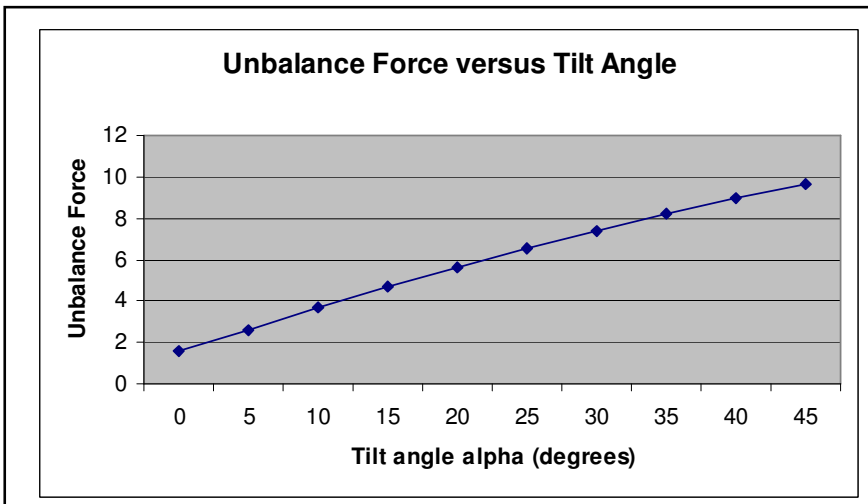

Figure 3: Graph of $f_{b}$ for $f_{w}=f_{r}=10$ and $\beta=45^{\circ}$

Fig. 3 assumes that the reaction mass is directly over the retracted leg. As the reaction mass rotates, the component of $f_{r}$ directly over the leg varies proportional to the cosine of the angle between the reaction mass and the retracted leg (Fig 4).

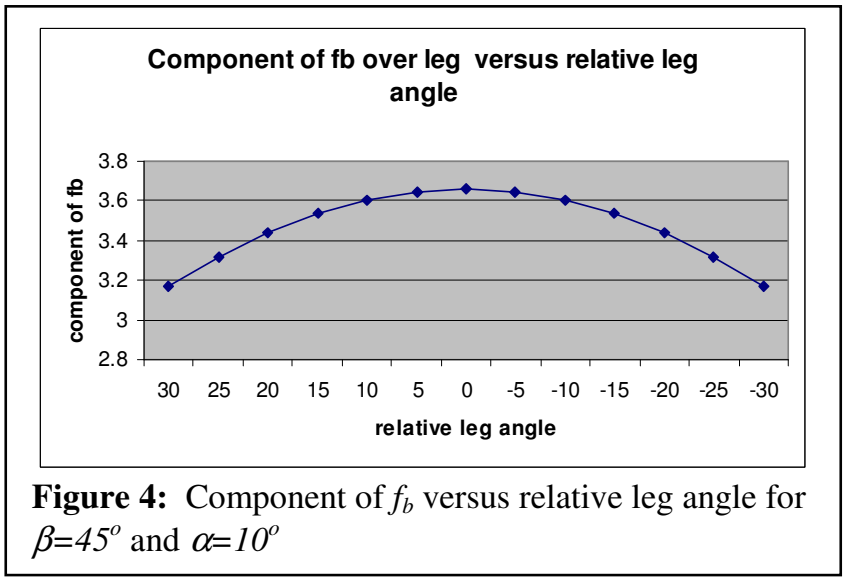

Once unbalanced, the mechanism will be supported only by the endpoint of the retracted leg. There is no longer any ground support to provide a reaction torque against the rotating mass, and the platform will rotate around the endpoint of the retracted leg $l_{i} \quad i \in\{1,2,3\}$ an amount $\theta_{i}$. We will model 
this angle as proportional to the time the legs are off the ground $t_{i}$ and the rotational velocity of the reaction mass, $\omega$ :

$$
\theta_{i}=\eta \omega t_{i}
$$

where $\eta$ is an attenuation constant of proportionality. By extending the leg, $M_{n e t}$ can be restored to zero, and the raised legs will drop down. The distance moved by the center is given by

$$
p_{i}=2 \ell_{e} \operatorname{Sin} \beta \operatorname{Sin} \theta_{i} / 2
$$

The center point moves in line segments of length $p_{i}$.

\section{Gaits}

Having constructed the model for stepping, we will now consider how the mechanism can locomote in a directed fashion. We will use the term gait to refer to a periodic step (i.e., $\left.\theta_{i}\right)$ pattern.

C.1 Constant-angle gaits. The plan view in Fig. 5 (a) shows a simulation rendering of the path the mechanism will follow if it is repeatedly stepped 30 degrees around each leg in turn i.e., a constant angle gait.

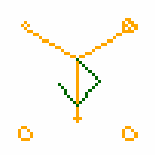

(a) $30^{\circ}$

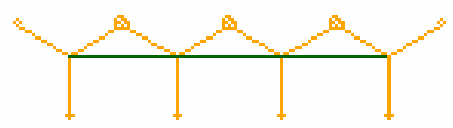

(b) $120^{\circ}$
Figure 5: Constant Angle Gaits

The initial position of the legs is shown in both figures. Subsequent positions of the legs are shown for the second figure, but not for the first (so that the view is not obscured). The tip of the leg around which the rotation occurs is shown as a small circle, and the dark line indicates the path described by the center.

If the step size is $120^{\circ}$ (Fig. 5(b)) then the mechanism will proceed in a straight line. This suggests a straightforward control scheme: Stepping at $120^{\circ}$ produces a straight line, a subsequent step of $120^{\circ}-\gamma$ will deviate $\gamma$ degrees left and a step of $120^{\circ}+\gamma$ will deviate $\gamma$ degrees right. This scheme produces a very agile robot, capable of producing very tight turns.

Fig. 6 shows a simulation rendering of the mechanism following a path around a right-hand and left-hand square.

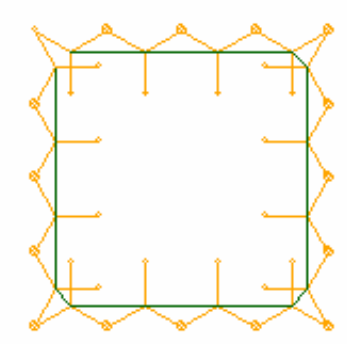

(a) right-hand

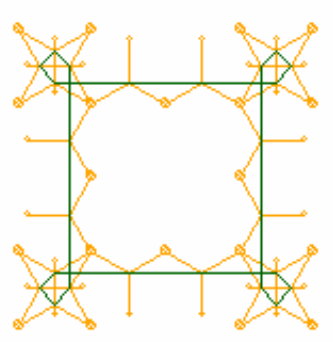

(b) left-hand
Figure 6: Simulated path around a square.
When a sequence of $120^{\circ}$ rotations is interrupted by a $30^{\circ}$ rotation, the mechanism makes a $90^{\circ}$ right-hand turn as shown in Fig. 6(a). A $90^{\circ}$ left-hand turn is accomplished by actually making a $270^{\circ}$ right-hand turn with three $30^{\circ}$ rotations, as illustrated in Fig. 6(b).

C.2 Repetitive constant angle gaits. This approach requires that the mechanism be capable of stepping arbitrary angles. We believe that this is ultimately possible for a general rotopod; however, it is certainly not possible for the prototype we have constructed (Section IV). The step size in the prototype is limited by design issues and by safety issues to at most $10^{\circ}$. One simple approach is to repeatedly step the mechanism around a single leg until the desired angle is reached (i.e., 12 times with step size $10^{\circ}$ to get $120^{\circ}$ and then repeated the process for each successive leg. We call this approach a repetitive constant angle gait. This slows the mechanism down by the factor of repetition (i.e., 12 above) but it allows for straight-line locomotion.

\section{C.3 Curve and Spiral Locomotion. If constant small angle} gaits are used, then straight line motion is not possible.

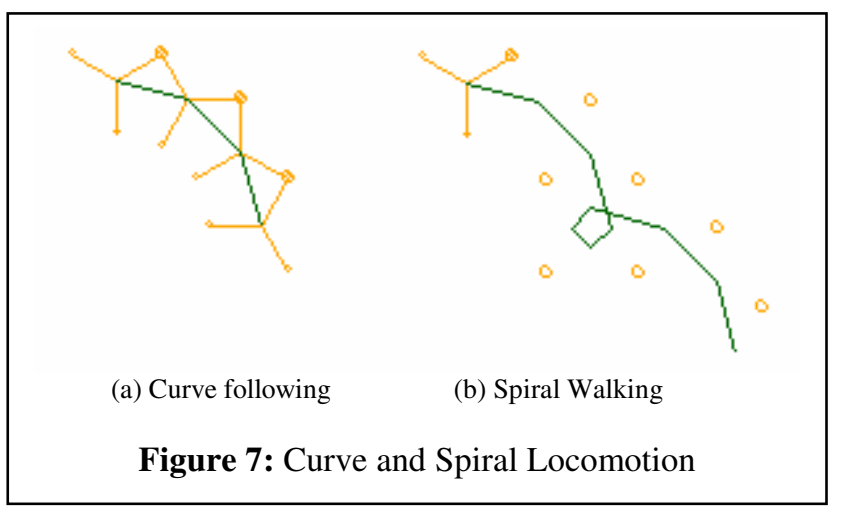

The center of the rotopod describes a curve when a repetitive $90^{\circ}$ gait is used, as shown in Fig. 7(a). This allows the rotopod to move to a specific location by selecting a set of radii of curvature.

Directional locomotion can be accomplished with a prolate cyloid path, as shown in Fig. 7(b). Here, the rotopod travels in the curve produced by a repetitive $90^{\circ}$ gait, with periodic $270^{\circ}$ turns (achieved by three successive rotations of $30^{\circ}$, one per leg) to adjust direction. Note that only the initial positions of the legs are shown in Fig. 7(b). Subsequent leg positions are not shown so that the view is not obscured. The small circles are still shown; they show the positions of the tips of the legs around which rotation occurs.

\section{Energy-efficient locomotion.}

To illustrate the potential energy efficiency of this method, we will compare its locomotion with that of a single wheel. In its most efficient mode, a tripedal rotopod would only use the unbalancing strategy to get two legs into the air, and at that point it would rotate like a wheel at the tilt angle to the ground plane. The legs would then be spokes of length $\ell \operatorname{Cos} \beta$ in a virtual wheel. Controlling such a mode of locomotion is not trivial as $\mathrm{Xu}$ et al. [14] demonstrate. Our current prototype could not be driven in this mode, but our goal is to build a 
prototype that can be. If we travel a distance $2 \pi \ell \operatorname{Cos} \beta$ in one time unit, then the specific resistance is

$$
\varepsilon_{w}=\frac{P(2 \pi \ell \operatorname{Cos} \beta)}{m g(2 \pi \ell \operatorname{Cos} \beta)}
$$

where $P()$ is the power used. This is the best case - a wheel. If we consider that our mechanism takes $3 k$ (for $k>1$ ) steps to travel this same distance, then instead of one rotation, the reaction mass will do $k$ rotations, using $k$ times the power. In addition, on each of the three steps per rotation, there is an extra energy term $P_{l}$ for the lowering of the leg.

$$
\varepsilon_{r}=\frac{k P(2 \pi \ell \operatorname{Cos} \beta)+3 P_{l}}{m g(2 \pi \ell \operatorname{Cos} \beta)}
$$

The ratio tells us how close we are to the best locomotion mode (where $\left.P_{w}=P(2 \pi \ell \operatorname{Cos} \beta)\right)$ :

$$
\frac{\varepsilon_{w}}{\varepsilon_{r}}=\frac{1}{k+3 P_{l} / P_{w}}
$$

\section{PROTOTYPE DESCRIPTION}

The previous section described a general tripedal rotopod. A rotopod mechanism can have more than three legs, and its leg actuation can be any system that allows unbalancing. We selected a simpler rotopod design for our first prototype.

\section{A. Prototype Mechanism}

The prototype is a tripedal system with a rotational knee joint in each leg. The prototype is shown in Figs. 8 and 9 and the Table of parameter values is presented in Appendix A.
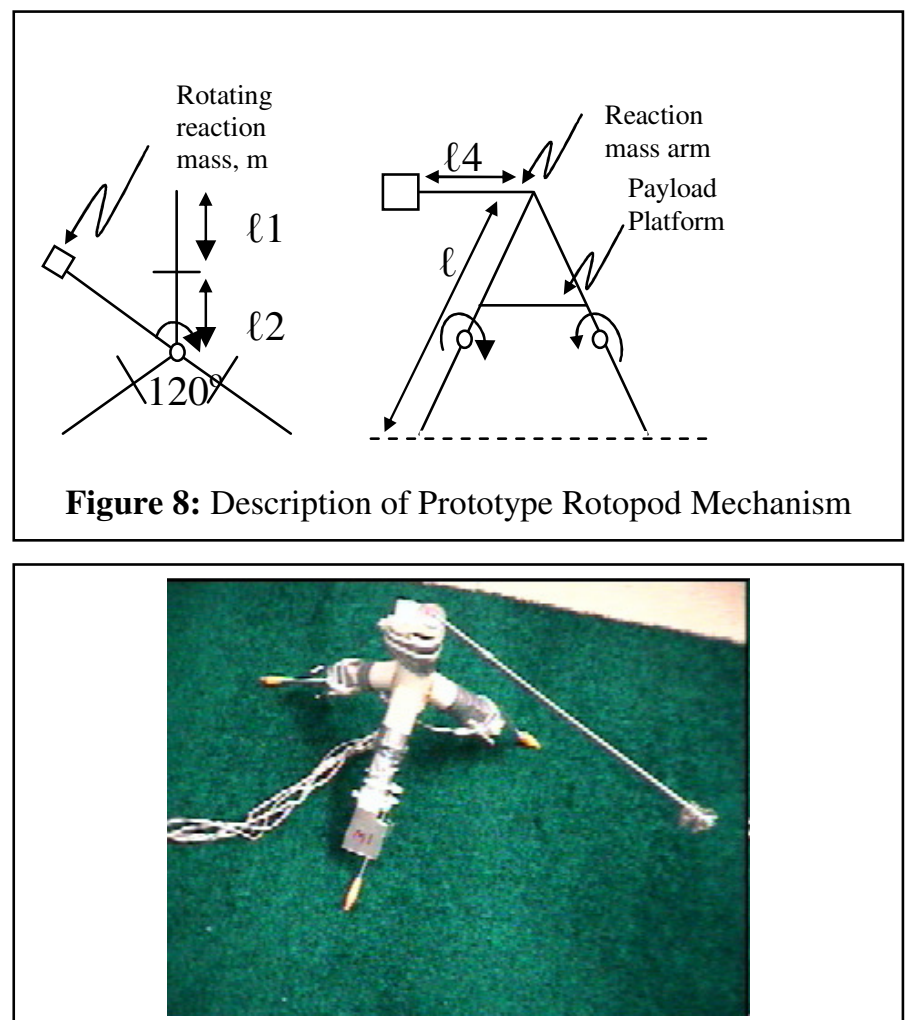

Figure 9: Prototype Rotopod Mechanism
Each of the three legs and the apex joint is equipped with a DC motor and gearbox. The lower legs are attached directly to the axle of each gearbox. There is a physical stop above and below each leg that limits the leg angle. To lower a leg, it is driven against the upper stop, a rotation of approx. $30^{\circ}$, while the other two legs are driven against their lower stop, straightening them to a rotation of $0^{\circ}$. The apex joint is driven with a constant rotational velocity of one rotation per second. This was established as a safe velocity.

\section{B. Control Hardware.}

The leg joints are driven from a Handyboard [10] using the bidirectional motor ports. The Handyboard was selected with the intention of eventually mounting it on the mechanism. For now, the Handyboard is separate, and the motor signals are fed from the board to the mechanism with an umbilical. The Handyboard is programmed using Interactive C (IC). No sensors are mounted on the prototype mechanism, and all actions are purely feed-forward, timed actions. We recognize that for more accurate motion, for faster motion, and for stabilizing the platform against unwanted unbalancing, sensors will be necessary.

\section{Control Software.}

The control scheme used to step the prototype is as follows. The one second rotation period of the reaction mass is divided into a duty cycle for each of the three legs. The duty cycle for a leg $l_{i}$ starts when the reaction mass is between the previous leg and this leg. When a leg is not in its duty cycle it is driven against the lower stop, so as to raise the leg to full length.

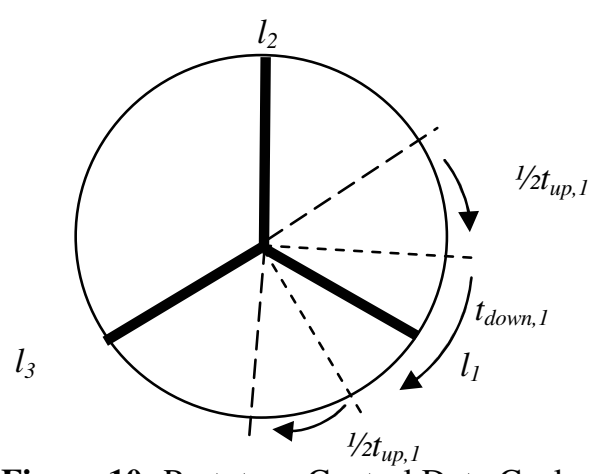

Figure 10: Prototype Control Duty Cycle

When a leg is within its duty cycle, for the first $1 / 2 t_{u p}$ seconds, the leg is maintained raised. For $t_{d o w n}$ seconds it is driven against the upper stop, lowering the leg. Finally, it is raised again for the last $1 / 2 t_{u p}$ seconds of the duty cycle. If $T$ is the period of the reaction mass, then

$$
\begin{gathered}
0.3 T=t_{\text {up }, i}+t_{\text {down }, i} \\
d_{i}=t_{\text {down }, i} / t_{\text {up }, i}
\end{gathered}
$$

The ratio $d_{i}$ is controlled for each leg. Our assumption is that the larger it is, the larger the step angle of the mechanism (up to a maximum of about $10^{\circ}$ for our prototype). We verify this assumption in the next section. 


\section{RESULTS}

In this section we report the results of a series of experiments to characterize the duty cycle model of stepping. The rotopod was placed beneath an overhead camera, and a sequence of video images was taken as the rotopod was repeatedly stepped around a single leg for a range of values of $d_{i}$. The video images were analyzed by hand to extract the position of the apex and endpoint of lowest leg.
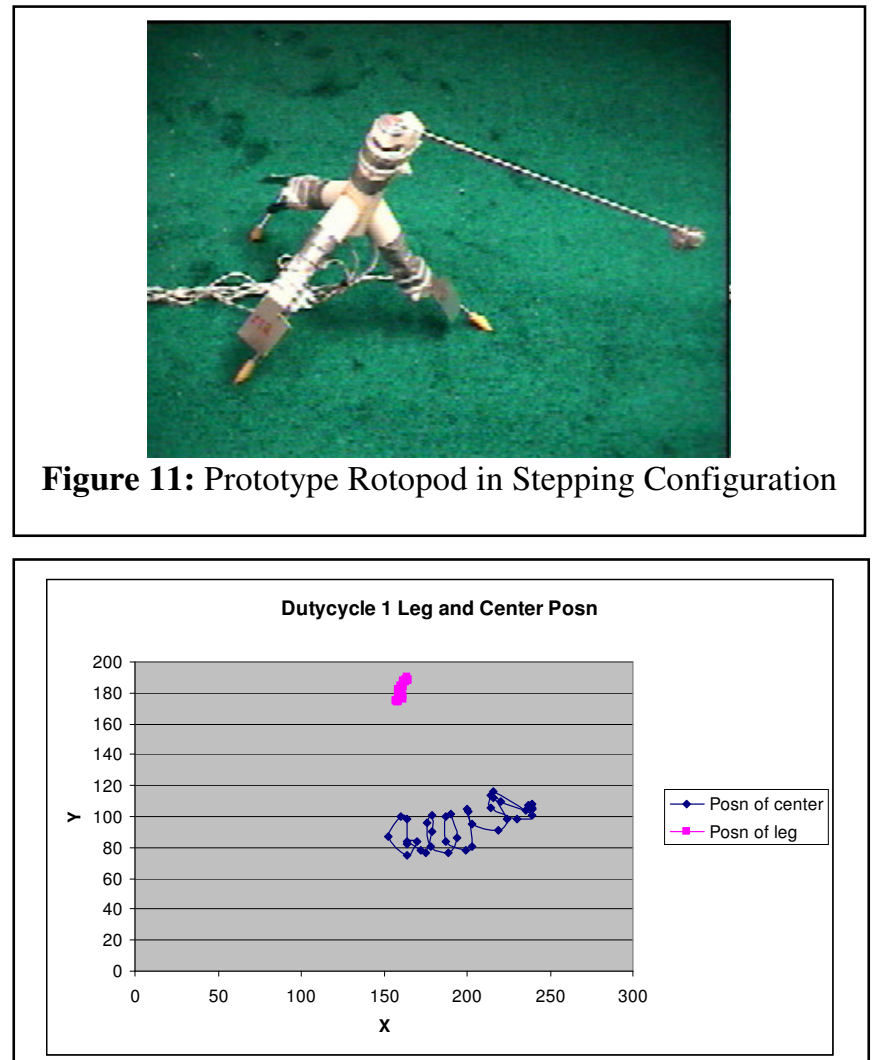

Figure 12: Graph of leg and center positions for seven steps of duty cycle $1(=0.6)$

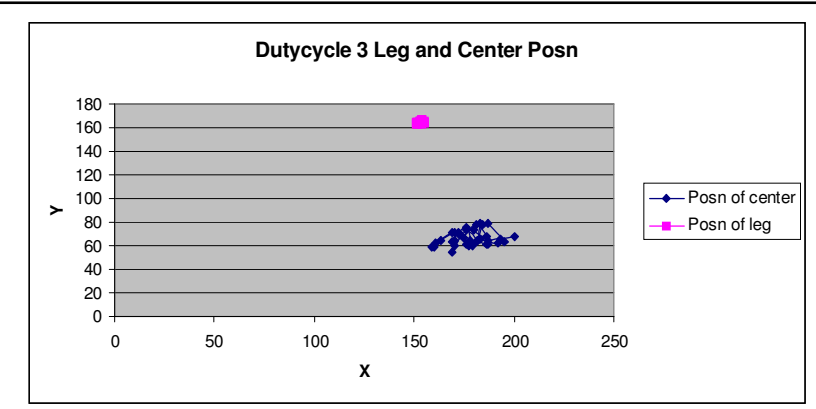

Figure 13: Graph of leg and center positions for seven steps of duty cycle $3(=0.25)$

The duty cycle was varied according to the values

$$
d_{i}=0.6,0.43,0.25,0.11
$$

In each case, the mechanism was stepped 7 times around the same leg $i$. The graphs for the duty cycles 0.6 and 0.25 are shown in Figure 12 and Figure 13 above. For the first duty cycle, the total change in orientation measured was $51^{\circ}$. The average step size was $7.28^{\circ}$ with a standard deviation of $2.41^{\circ}$.

The unevenness in successive rotations has a component of measurement error due partly to variable video recording timing, and due partly to human observation error. There is a component of modeling error, based on use of the duty cycle model and the (manual) synchronization of legs and reaction mass motion. The final component is due to the inaccuracies in the prototype construction.

The epicyclical path of the center (Fig. 12) is due to uncontrolled leg bending as the reaction mass passed over each leg. To combat this issue, the position of the reaction mass needs to be known, so as to stiffen the legs (see final section) and prevent unwanted motion.

Notice also that there is translation of the endpoint of the lowest leg - ideally there should just be a rotation. The translation also appears to be proportional to the duty cycle.

For the third duty cycle, the average step size was $3.7^{\circ}$ and the standard deviation was $1.6^{\circ}$. Figure 14 shows a plot of all four duty cycles against their average measured step size.

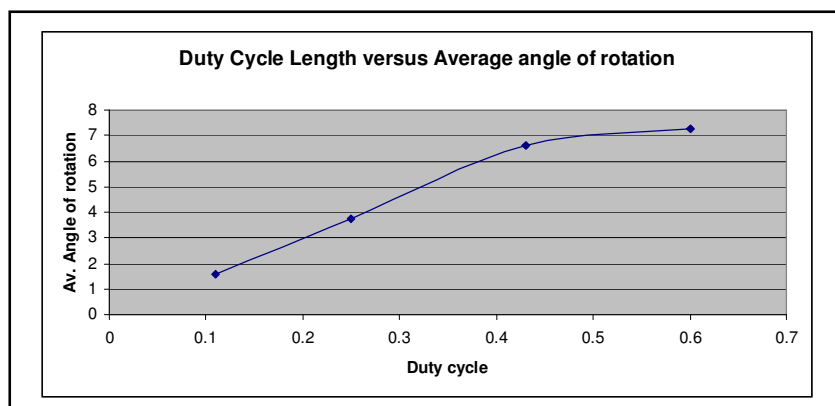

Figure 14: Duty cycle versus angle of rotation

To approximate a value for the attenuation $\eta$ we need to know how long the legs were off the ground for each duty cycle. This was not measured, however, we can approximate that at most the legs were off the ground for the $t_{\text {down }}$ component of the duty cycle. This gives an average smallest value of $\eta=0.55$.

\section{CONCLUSIONS}

In this paper we have introduced a new model of locomotion that has the potential for very agile and efficient motion. Our prototype results indicate that that the mechanism can be made to operate, albeit in a relatively slow fashion due to the limitations of our prototype. We don't believe any of these limitations are insurmountable, but they leave a number of areas of future work.

1. Sensor use. The current control scheme uses no sensor feedback, and is limited to no more than 5 to 10 steps in succession before timing inaccuracies cause the duty cycle in the legs and rotating mass to go out of synchronization. If the angle of rotation of the mass was known, it could be used to keep the legs 
in synchronization.

A gyroscope sensor would allow the tilt of the base to be measured and used as feedback for leg tilt. When the rotopod is deployed on rough terrain, this sensor will be essential.

2. Improved mechanism. The rotopod frame is very simple - basically a tetrahedral frame with legs on 3 vertices and the reaction mass motor on the $4^{\text {th }}$. Translational, spring loaded leg joints would be a better configuration than the rotational joints of the prototype. The latter had the advantage of easy availability for fast construction of the prototype.

In addition, if the leg motors were replaced with DC servo motors, then a more precise manner of control could be implemented than the leg control scheme in the prototype. This would also be useful for handling some of the undesirable, unbalancing forces exerted on the mechanism as the mass rotates. Smaller, controlled leg motions can be used to stabilize the base against these forces (at the cost of a higher power usage, of course).

3. On-board control. The Handyboard was selected with the ultimate aim of mounting on the prototype. We believe this is quickly possible, but we also believe a power umbilical will be necessary until the additional battery weight can be handled.

4. Recovery from overturning. When the mechanism is on its side, due to falling over for example, then it is possible to envision a series of actions that can take it back to an upright position. The reaction mass arm can be used as a 'leg' to lever the tetrahedral frame, and it is possible that together with synchronized leg motions, this can be used to right the mechanism.

5. Navigation. The rotopod has an unusual style of locomotion, and further work is required to develop an efficient path planning approach. The style of locomotion may be especially appropriate for applications that demand the path have some guaranteed properties of a space-filling curve.

\section{REFERENCES}

[1] Altendorfer, R., Moore, E. Z., Komsuoglu, H., Buehler, M., Brown Jr., H. B., McMordie, D., Saranli, U., Full, R., Koditschek, D. E., RHex: A Biologically Inspired Hexapod Runner, Autonomous Robots 11:207-213, 2001.

[2] Angles, C. and Brooks, R., Small Planetary Rovers. IEEE Workshop on Intelligent Robots and Systems, 1990.

[3] Bares, J., and Wettegreen, D., Dante II, IJRR V18, N7 1999.pp.621-649.

[4] Badreddin, E., (Automation Laboratory University of Mannheim.) and Gruener, G., (ETH Zurich) http://www.proaut.uni-

mannheim.de/research/projects/3_legged_robot.htm

[5] Bevly, D., Farritor, S., Dubowsky, S., Action planning module and its application to an experimental climbing robot. IEEE Int. Conf. on Robotics and Automation, San Francisco, CA, Vol. 4, pp. 4009-4014, April 2000.
[6] Collins, S. H., Ruina, A. (2005) A bipedal walking robot with efficient and human-like gait. To appear: Proc. IEEE Int. Conf. on Rob. and Aut., Barcelona, Spain, April 2005

[7] Halme, A., Leppänen, I., Montenon, M. and Ylönen, S. Robot Locomotion by Simultaneous Wheel and Leg Propulsion. CLAWAR'2001, Karlsruhe Germany 2001.

[8] Heng K.H., Zielinska, T., Multifunctional Walking Quadruped, Robotica Vol. 20, pp 585 - 593, 2002.

[9] H. Itto and K. Fukuda, Yamagata University. http://133.24.70.46/english/robots.shtml.

[10] Martin, F., Robotic Explorations: A Hands-on Introduction to Engineering. Prentice-Hall 2001.

[11] Quinn, R. D. Nelson, G.M., Bachmann, R.J., Kingsley, D.A., Offi, J. and Ritzmann, R. E., Insect Designs for Improved Robot Mobility. Proc. of Climbing and Walking Robots Conf. (CLAWAR01), Karlsruhe, Germany, pp. 6976, 2001.

[12] Raibert, M., Legged Robots that Balance. MIT Press 1986.

[13] Raibert, M. H., Chepponis, M., Brown, H. B. Jr. 1986. Running on four legs as though they were one. IEEE $J$. Robotics and Automation, 2:70--82.

[14] $\mathrm{Xu}, \mathrm{Y}$., and $\mathrm{Ou}$, S.K.W., Stabilization and path following of a single wheel robot, IEEE/ASME Transactions on Mechatronics, Vol. 9, No. 2, pp. 407-419, June 2004

\section{Appendix A: Table of Prototype Parameter Values}

\begin{tabular}{|l|l|l|}
\hline Name & Description & Value \\
\hline$\ell 1$ & Length of lower leg & $10.2 \mathrm{~cm}$ \\
\hline$\ell 2$ & Length of upper leg & $10.2 \mathrm{~cm}$ \\
\hline$\ell 3$ & Vertical offset of reaction mass joint & $8.9 \mathrm{~cm}$ \\
\hline$\ell 4$ & Length of reaction mass arm & $30.5 \mathrm{~cm}$ \\
\hline$\ell_{\mathrm{e}}$ & Extended Leg length $(\ell 1+\ell 2)$ & $20.3 \mathrm{~cm}$ \\
\hline & Min/Max angle of leg bend & $0^{\circ} / 30^{\circ}$ \\
\hline$\beta$ & Angle between leg and centerline & $45^{\circ}$ \\
\hline $\mathrm{m}_{\mathrm{r}}$ & Reaction mass & $30 \mathrm{~g}$ \\
\hline $\mathrm{m}_{\mathrm{v}}$ & Vehicle mass without $\mathrm{m}_{\mathrm{r}}$ & $430 \mathrm{~g}$ \\
\hline $\mathrm{m}$ & Total mass $\left(\mathrm{m}_{\mathrm{r}}+\mathrm{m}_{\mathrm{v}}\right)$ & $460 \mathrm{~g}$ \\
\hline
\end{tabular}

\section{Appendix B: Terminology}

\begin{tabular}{|l|l|}
\hline Name & Description \\
\hline$\ell$ & Retracted leg length \\
\hline$\delta$ & Leg extension \\
\hline$\ell_{\mathrm{e}}$ & Extended leg length, $\ell+\delta$ \\
\hline$\beta$ & Angle between leg and centerline \\
\hline $\mathrm{f}$ & Distance from retracted to adjacent leg \\
\hline$\alpha$ & Angle of platform tilt due to retracted leg \\
\hline$t_{i}$ & Time leg $i$ is retracted \\
\hline$\omega$ & Angular velocity of reaction mass \\
\hline$\theta_{\mathrm{i}}$ & Angle base rotates around leg $i$ \\
\hline$\eta$ & Attenuation constant \\
\hline$\varepsilon$ & Specific resistance \\
\hline
\end{tabular}

